\title{
Degradación Fotocatalítica de Contaminantes Orgánicos Presentes en Agua Residual de la Industria Litográfica mediante Oxido de Bismuto(III)/Oxido de Titanio $\left(\mathrm{Bi}_{2} \mathrm{O}_{3} / \mathrm{TiO}_{2}\right)$
}

\author{
Andrés López-Vásquez ${ }^{(1,2)}$, Ovidio Almanza ${ }^{(3)}$, Manuel F. Acosta ${ }^{(3)}$ y Andrés Suárez-Escobar ${ }^{(4)}$ \\ (1) Departamento de Ingeniería Ambiental, Universidad Libre-Sede Bogotá. Colombia. \\ (2) Escuela de Ingeniería Química, Universidad del Valle, Cali, Colombia. \\ (3) Departamento de Física, Grupo de Física Aplicada, Universidad Nacional de Colombia-Sede Bogotá, \\ (4) Departamento de Ingeniería Química, Universidad de Bogotá Jorge Tadeo Lozano. Bogotá-Colombia. \\ (e-mail: andresf.lopez@unilibrebog.edu.co;oaalmanzam@unal.edu.co;mafacostahu@unal.edu.co; \\ andresf.suareze@utadeo.edu.co)
}

Recibido Jul. 3, 2015; Aceptado Ago. 28, 2015; Versión final Oct. 14, 2015, Publicado Feb. 2016

\begin{abstract}
Resumen
Se prepararon semiconductores acoplados de $\mathrm{Bi}_{2} \mathrm{O}_{3} / \mathrm{TiO}_{2}$ mediante el método de impregnación húmeda. La actividad fotocatalítica se evaluó sobre la degradación de compuestos orgánicos presentes en agua residual generada por la industria litográfica bajo irradiación UV-visible y fueron caracterizados mediante difracción de rayos $x$, espectroscopia UV visible e infrarroja. Se utilizó la metodología de superficie de respuesta a través de un diseño factorial de tres niveles para evaluar el efecto combinado de la relación $\mathrm{Bi}_{2} \mathrm{O}_{3} / \mathrm{TiO}_{2}$ y el pH inicial de la solución. Las respuestas evaluadas fueron los porcentajes de remoción de carbono orgánico total y Demanda Química de Oxígeno. Los resultados revelaron que los materiales compuestos (composites) de $\mathrm{Bi}_{2} \mathrm{O}_{3} / \mathrm{TiO}_{2}$ mostraron un menor tamaño de cristal, mayor estabilidad térmica y una mayor absorción en el rango visible que el $\mathrm{TiO}_{2}$ puro
\end{abstract}

\section{Photocatalytic Degradation of Organic Pollutants from Printing Wastewater by Bismuth(III) Oxide/Titanium Oxide $\left(\mathrm{Bi}_{2} \mathrm{O}_{3} / \mathrm{TiO}_{2}\right)$}

\begin{abstract}
Coupled semiconductors of $\mathrm{Bi}_{2} \mathrm{O}_{3} / \mathrm{TiO}_{2}$ were synthetized by wet impregnation. Photocatalytic activity was evaluated upon the removal of organic pollutants presents in lithographic wastewater under UV-vis radiation. Catalyst were characterized by $\mathrm{X}$ ray diffraction, diffuse reflectance spectroscopy and Fourier transform infrared spectroscopy. Response surface methodology was employed with two factors in three to evaluate the combined effect of the ratio $\mathrm{Bi}_{2} \mathrm{O}_{3} / \mathrm{TiO}_{2}$ and the initial $\mathrm{pH}$ of the solution. The responses evaluated were the removal percentages of total organic carbon and chemical oxygen demand. The results showed that $\mathrm{Bi}_{2} \mathrm{O}_{3} / \mathrm{TiO}_{2}$ composites have smaller crystal size, higher thermal stability and a higher absorption in the visible range than pure $\mathrm{TiO}_{2}$.
\end{abstract}




\section{INTRODUCCIÓN}

En la industria litográfica se genera una amplia variedad de residuos y efluentes asociados al desarrollo de etapas tales como el procesamiento de imágenes, diseño de plantillas, impresión y acabado. Estos son producidos principalmente por residuos de papel, envases vacíos de tintas, restos de solventes y emisiones gaseosas (tintas y solventes) conocidas como compuestos orgánicos volátiles (COV's). Particularmente, el agua residual originada durante el proceso se caracteriza por ser una compleja mezcla de compuestos orgánicos e inorgánicos, en cuyo contenido se pueden encontrar restos de solventes que pueden ser inflamables o combustibles, pigmentos que le dan una coloración excesiva y elevados valores de pH debido a la presencia de productos detergentes utilizados en la limpieza de plantillas, rodillos y prensas. También se pueden encontrar restos de grasas y aceites e incluso adhesivos que contienen componentes que interrumpen la acción biológica en los tratamientos de aguas residuales [Jendrucko, R. J. et al., 1994]. Este efluente constituye un riesgo ambiental, por lo que debe ser tratado antes de ser descargado a la fuente natural, pero debido a la característica de sus componentes (recalcitrantes) tales como toxicidad y bajo índice de biodegradabilidad, hacen que la implementación de una estrategia biológica de tratamiento sea una opción prácticamente inviable.

La fotocatálisis heterogénea con dióxido de titanio $\left(\mathrm{TiO}_{2}\right)$ es una técnica no selectiva con la que se puede degradar compuestos contaminantes mediante oxidación avanzada. Esta ha sido probada en el tratamiento de este tipo de aguas residuales pero uno de los principales inconvenientes se debe a que debido al ancho de banda del semiconductor (3.2 eV), sólo aprovecha el 5.0\% del espectro solar (radiación UV) para llevar a cabo el proceso fotocatalítico. Con el fin de aumentar su actividad, una de las estrategias es la generación de nuevas especies oxidantes bajo el rango visible y evitar la recombinación de par electrón/hueco, fenómeno indeseable en los procesos fotocatalíticos. Lo anterior se puede lograr a través del dopado con metales de transición u otro elemento dopante, y el uso de semiconductores acoplados (lkeda et al., 2003; lliev et al., 2003; Gurunathan, 2004). Ejemplos de sistemas semiconductores acoplados que han sido utilizados para tal fin son $\mathrm{CdS} / \mathrm{TiO}_{2}$ ( $\mathrm{Li}$ et al., 2010), $\mathrm{ZnO} / \mathrm{TiO}_{2}$ (Ge et al., 2009), $\mathrm{SnO}_{2} / \mathrm{TiO}_{2}$ (Hou et al., 2007), $\mathrm{SiO}_{2} / \mathrm{TiO}_{2}$ (Cetinkaya et al., 2013), $\mathrm{Fe}_{2} \mathrm{O}_{3} / \mathrm{TiO}_{2}$ (Banisharif et al., 2015), Galeano et al., 2013, entre otros, y mostraron adecuados niveles de degradación de varios compuestos orgánicos. El óxido de bismuto $\left(\mathrm{Bi}_{2} \mathrm{O}_{3}\right)$, es un material semiconductor con un ancho de banda directo de $2.8 \mathrm{eV}$ que puede ser excitado por luz visible pero su baja actividad fotocatalítica se debe a la fotocorrosión y a la rápida recombinación del par electrón/hueco fotogenerado en su superficie (Hameed et al., 2009). Debido a sus propiedades, se ha investigado el acople con $\mathrm{TiO}_{2}$ con el fin de desarrollar un sistema que pueda ser activado bajo irradiación visible y además sea estable fotoquímicamente. El principal desarrollo ha girado en torno al método de preparación por lo que se han probado técnicas tales como el autoensamblaje por evaporación inducida (EISA), el proceso hidrotérmico para conformación de nanopartículas, el reflujo ácido y el método sol-gel no acuoso para la dispersión del mismo. Estos métodos de síntesis han demostrado las bondades en la conformación estructural del sistema y su efecto sobre la actividad fotocatalítica, aunque los métodos de preparación no son sencillos y requieren esfuerzos técnicos que demandan en muchos casos, altos costos de inversión. Una opción podría ser la impregnación húmeda, la cual brinda la posibilidad de acoplar materiales de manera sencilla y económica mediante activación térmica para conformar compósitos.

Aunque se han desarrollado estudios de actividad fotocatalítica utilizando este sistema acoplado (Yuande et al., 2010), la mayoría de éstos se han llevado a cabo variando un factor a la vez y manteniendo los demás constantes. Esta metodología de optimización es inadecuada debido a que no considera el efecto combinados de los parámetros involucrados en el proceso además, os procesos fotocatalíticos suponen una compleja interacción entre el sustrato, catalizador y la interacción con la luz incidente, el modelado de la operación presenta cierto grado de complejidad, en situaciones en las que el sistema de reacción es una mezcla heterogénea de contaminantes. Una de las estrategias utilizadas es el modelado estadístico mediante la metodología de superficie de respuesta (MSR), la cual es una técnica que combina la matemática y la estadística para evaluar la relativa significancia de los diferentes factores del proceso, aun en sistemas complicados. La ventaja del método es la determinación de las condiciones óptimas de operación, mediante la reducción del tiempo y costo del análisis (Ohno et al., 2003). En esta investigación, se evaluó el efecto de las variables relación $\mathrm{Bi}_{2} \mathrm{O}_{3} / \mathrm{TiO}_{2}$ y pH inicial sobre la remoción de carga orgánica contaminante (COT y DQO) presente en una muestra real de agua residual proveniente de la industria litográfica bajo irradiación UV-Vis artificial mediante la metodología de superficie de respuesta (factorial de 3 niveles con 4 puntos centrales) y se determinaron los parámetros cinéticos de la degradación.

\section{MATERIALES Y METODOS}

\section{Preparación del catalizador acoplado $\mathrm{Bi}_{2} \mathrm{O}_{3} / \mathrm{TiO}_{2}$}

La preparación del material fotocatalítico se llevó a cabo mediante el método de la impregnación húmeda. En un experimento típico, el soporte sólido $\left(\mathrm{TiO}_{2}\right.$ Degussa P-25, AEROXIDE $\left.{ }^{\circledR}\right)$ y la especie activa $\left(\mathrm{Bi}_{2} \mathrm{O}_{3}\right.$, 
Sigma Aldrich) se suspendieron en $5 \mathrm{ml}$ de $\mathrm{NH}_{4} \mathrm{OH} 2.5 \%$ (Carlo Erba) y $50 \mathrm{ml} \mathrm{H}_{2} \mathrm{O}_{2} 30 \%$ (Merck), bajo fuerte agitación magnética a temperatura ambiente. Las cantidades de los óxidos fueron las correspondientes para preparar composiciones de la fase activa de 5.0, 8.0, $25 \%$ en peso $(0.05: 1,0.08: 1 \mathrm{y}$ $0.25: 1)$, respectivamente. La suspensión amarillenta obtenida fue sonicada durante 180 min para promover una mezcla homogénea de los reactivos. El precipitado fue filtrado y lavado con agua desionizada (resistividad $=0.067 \mu \mathrm{S} \mathrm{cm}{ }^{-1}$ ) varias veces para eliminar el exceso de iones $\mathrm{NH}_{4}^{+}$y el sólido húmedo se secó a $110{ }^{\circ} \mathrm{C}$ durante $24 \mathrm{~h}$ con el fin de ser activado mediante calcinación a $300^{\circ} \mathrm{C}$ durante $3 \mathrm{~h}$. Para la degradación de aguas residuales de la industria litográfica, se tomó una muestra representativa de una industria local (830 ppm COT, 1978 ppm DQO y pH 8.83).

\section{Pruebas de caracterización}

La identificación de las fases cristalinas y las propiedades estructurales de los fotocatalizadores preparados, se llevó a cabo mediante difracción de rayos $X$ (XRD) (Panalytical X'Pert PRO con radiación KaCu, $\lambda=$ $1.540598 \AA$ ) a $2 \theta$ entre $15^{\circ}$ y $60^{\circ}$ (variación de ángulo de $0.02^{\circ}$ ). El espectro de reflectancia difusa (UV-Vis DRS) fue determinado en un espectrofotómetro Shimadzu UV-Vis $2600\left(\mathrm{BaSO}_{4}\right.$ fue utilizado como referencia) y la identificación de las vibraciones de enlace, se determinó mediante el espectroscopía infrarroja a temperatura ambiente (FT-IR Prestige 21, Shimadzu) en el rango de $4000-400 \mathrm{~cm}^{-1}$.

\section{Experimentos fotocatalíticos}

El sistema óptico utilizado para la degradación fotocatalítica de contaminantes orgánicos presentes en aguas residuales de la industria litográfica, constó de una lámpara de Xe de $20 \mathrm{~W}$ ubicada a $10 \mathrm{~cm}$. sobre la superficie de la suspensión reaccionante, la cual además de emitir radiación en el espectro visible, presentó una $\lambda_{\max }, \mathrm{UV}=365 \mathrm{~nm}$ (Figura 1). Para cada experimento, $0.125 \mathrm{~g}$ de compósito fueron adicionados a $250 \mathrm{~mL}$ de agua residual y transferidos a un vaso de precipitado de $300 \mathrm{~mL}$ el cual se usó como reactor. Antes de iniciar la irradiación, la suspensión fue agitada en la oscuridad durante $30 \mathrm{~min}$ favoreciendo el equilibrio de adsorción (en este punto, la concentración del agua residual fue considerada como valor inicial). Durante la irradiación, la suspensión se agitó magnéticamente y la temperatura de reacción se mantuvo en $25^{\circ} \mathrm{C}$ mediante un baño termostatado. Luego de $5 \mathrm{~h}$ de tratamiento, se tomaron de $5 \mathrm{~mL}$ de muestra y se pasaron a través de un filtro de membrana (Nylon $0.45 \mu \mathrm{m}$ ) con el fin de remover el catalizador en suspensión. El filtrado fue analizado para determinar el contenido de COT (Analizador TOC-L, Shimadzu) y la DQO (SMEWW-5220) presente en la muestra tratada.

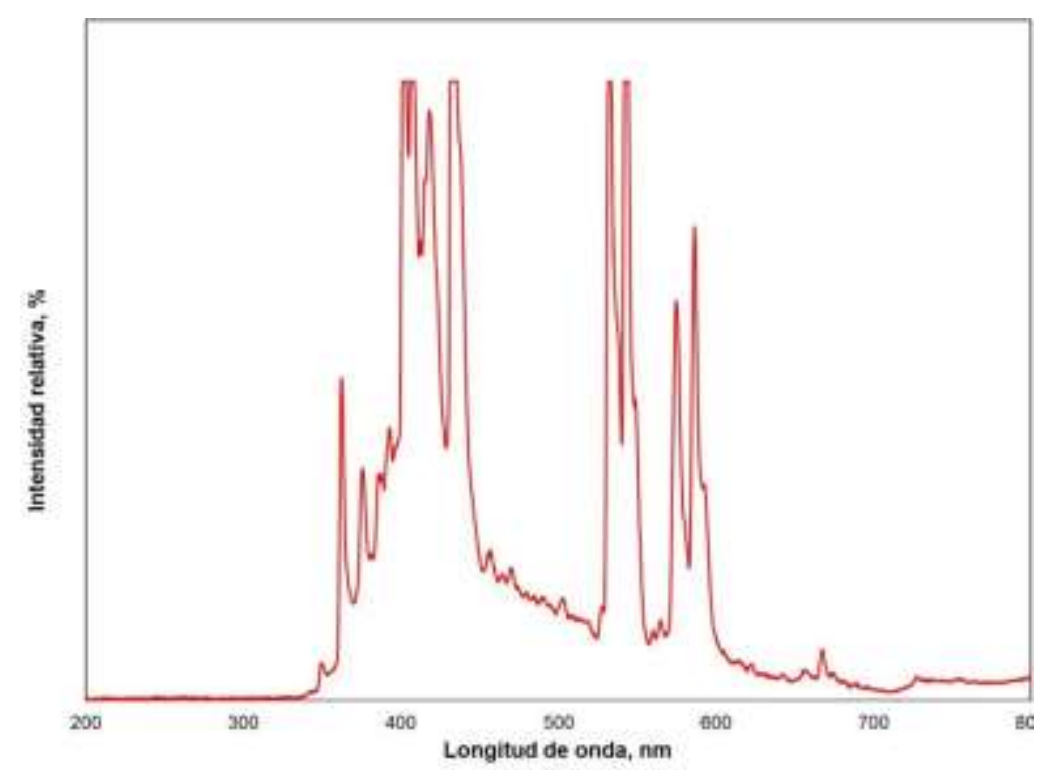

Fig. 1. Espectro de emisión de la lámpara de Xe utilizada en los ensayos de degradación fotocatalítica de contaminantes orgánicos presentes en agua residual de la industria litográfica.

\section{Diseño experimental}

La metodología de superficie de respuesta (MSR) se utilizó para optimizar las variables relación $\mathrm{Bi}_{2} \mathrm{O}_{3} / \mathrm{TiO}_{2}$ $(0.05: 1$ - 0.25:1) y el pH inicial $(5.0$ - 9.0). Estos parámetros se seleccionaron como variables independientes mientras que los porcentajes de degradación COT y DQO, fueron las variables de respuesta. 
Tabla 1. Diseño central compuesto con resultados experimentales y predichos de la degradación fotocatalítica de contaminantes orgánicos presentes en agua residual de la industria litográfica.

\begin{tabular}{|c|c|c|c|c|c|c|}
\hline \multirow[b]{3}{*}{ Corrida } & \multirow{2}{*}{\multicolumn{2}{|c|}{$\begin{array}{c}\text { Condiciones } \\
\text { experimentales }\end{array}$}} & \multicolumn{4}{|c|}{ Porcentaje de remoción, \% } \\
\hline & & & \multicolumn{2}{|c|}{ COT } & \multicolumn{2}{|c|}{$D Q O$} \\
\hline & $\begin{array}{c}x_{1}, \text { Relación } \\
\mathrm{Bi}_{2} \mathrm{O}_{3} / \mathrm{TiO}_{2}\end{array}$ & $x_{2,}, p H$ & Experimental & Predicho & Experimental & Predicho \\
\hline 1 & 0.08 & 7.00 & 16.2 & 15.85 & 19.3 & 18.9 \\
\hline 2 & 0.08 & 7.00 & 15.4 & 15.85 & 20.5 & 18.9 \\
\hline 3 & 0.08 & 5.00 & 33.3 & 35.66 & 32.6 & 36.66 \\
\hline 4 & 0.05 & 7.00 & 4.9 & 11.05 & 5.8 & 12.36 \\
\hline 5 & 0.08 & 7.00 & 15.8 & 15.85 & 20.2 & 18.9 \\
\hline 6 & 0.25 & 7.00 & 27.6 & 21.58 & 24.9 & 20.63 \\
\hline 7 & 0.25 & 9.00 & 47.8 & 51.15 & 35.2 & 37.77 \\
\hline 8 & 0.08 & 7.00 & 15.9 & 15.85 & 18.1 & 18.9 \\
\hline 9 & 0.08 & 7.00 & 16.1 & 15.85 & 18.7 & 18.9 \\
\hline 10 & 0.08 & 9.00 & 39.1 & 36.87 & 32.7 & 30.93 \\
\hline 11 & 0.05 & 9.00 & 31.7 & 30.57 & 24.3 & 23.49 \\
\hline 12 & 0.25 & 5.00 & 30.2 & 32.86 & 31.6 & 33.29 \\
\hline 13 & 0.05 & 5.00 & 37.4 & 32.37 & 36.8 & 31.03 \\
\hline
\end{tabular}

Factores tales como velocidad de agitación e intensidad de radiación permanecieron constantes. Se eligió un diseño factorial $2^{3}$ con 4 réplicas en el punto central (13 experimentos) para evaluar el efecto combinado de las variables independientes. Los valores experimentales de los porcentajes de degradación bajo diferentes condiciones experimentales se muestran en la Tabla 1. Se utilizó el software Design Expert V.7.0.0 (Stat-Ease Inc., Minneapolis, MN, USA) para describir la superficie y se comprobó la precisión del modelo ajustado mediante una serie de análisis estadístico tales como el gráfico normal, el análisis de residuales, la interacción de los efectos principales, los gráficos de contorno y el análisis de varianza.

Las cinéticas de remoción del contenido de COT y DQO para la degradación fotocatalítica de aguas residuales de la industria litográfica, se llevaron a cabo bajo las condiciones en las que se presentó los mayores porcentajes de remoción de COT y DQO (0.25:1 y pH 9.0).

\section{RESULTADOS Y DISCUSION}

\section{Caracterización de los acoples $\mathrm{Bi}_{2} \mathrm{O}_{3} / \mathrm{TiO}_{2}$}

La Figura 2 muestra los patrones XRD de las muestras con relación atómica $\mathrm{Bi}-\mathrm{Ti}, 0.05,0.08$ y 0.25:1, respectivamente. La fase cristalina anatasa presentada en las muestras acopladas (compósitos) está asociada a los picos de difracción característicos del $\mathrm{TiO}_{2}$, JCPDS 089-4921 (fase anatasa), localizados en las posiciones $2 \theta=25.25,37.88$ y 48.00, correspondientes a índices de Miller (101), (004) y (200) respectivamente. Para la fase $\mathrm{Bi}_{2} \mathrm{O}_{3}$, JCPDS 045-134, los picos de difracción ubicados entre $2 \theta=27.44^{\circ}$, $30.40^{\circ}$ y $32.8^{\circ}$ corresponden a planos con índices de Miller (310), (222) y (321), respectivamente. Se observa un pequeño solapamiento del pico de difracción correspondiente al plano (101) del $\mathrm{TiO}_{2}$ a mayores cantidades (0.08:1 y $0.25: 1 \quad \mathrm{Bi}_{2} \mathrm{O}_{3} / \mathrm{TiO}_{2}$ respectivamente), debido a que en posiciones $2 \theta$ similares se encuentran picos de difracción asociados al $\mathrm{Bi}_{2} \mathrm{O}_{3}$. En los demás picos de difracción asociados al $\mathrm{TiO}_{2}$, no se observaron desplazamientos en la difracción (las principales fases presentes en la referencia Degussa P25 son la anatasa y rutilo, siendo la anatasa la fase mayoritaria, Suttiponparnit et al., 2011). De la misma manera, se determinó el porcentaje experimental de $\mathrm{Bi}_{2} \mathrm{O}_{3}$ presente en cada fotocatalizador acoplado $\left(\mathrm{Bi}_{2} \mathrm{O}_{3} / \mathrm{TiO}_{2}\right)$, el cual concordó con el porcentaje nominal de cada relación atómica de $\mathrm{Bi}-\mathrm{Ti}$ de cada compósito preparado.

La determinación del tamaño de cristal $D_{s}$ para el sistema $\mathrm{Bi}_{2} \mathrm{O}_{3} / \mathrm{TiO}_{2}$, se determinó mediante la ecuación de Scherrer (Ecuación 1).

$$
D_{s}=\frac{K \lambda}{\beta \cos \theta}
$$

Donde $\beta$ es la integral de amplitud del perfil intrínseco, $\lambda$ es la longitud de onda de los rayos $\mathrm{X}, K$ es la constante de Scherrer, cuyo valor es 0.94 y $\theta$ es el ángulo Bragg de cada pico de difracción. A su vez, $\beta$ puede ser escrita mediante la Ecuación 2, en donde $\beta_{\text {instr }}$ y $\beta_{m}$ son las contribuciones debido al instrumento y a las muestras, respectivamente (Waren, 1968). 


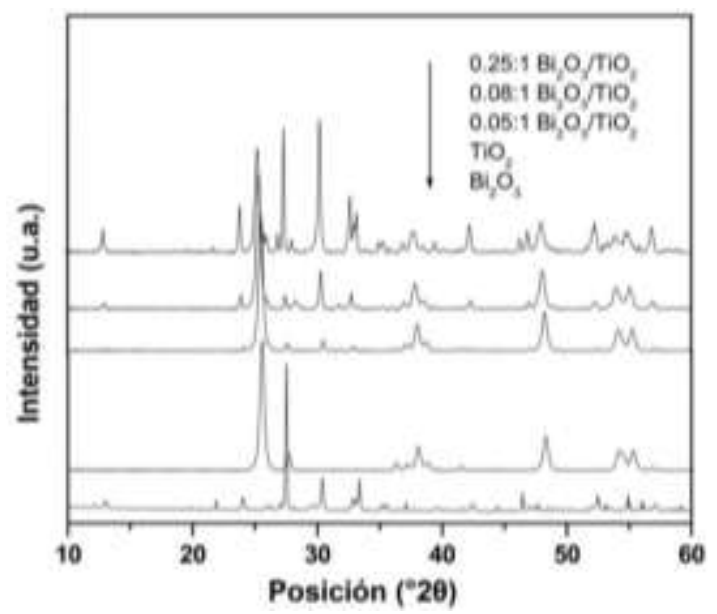

Fig. 2. Patrones XRD de las muestras con diferentes relaciones atómicas de $\mathrm{Bi}-\mathrm{Ti}$ calcinadas a $300^{\circ} \mathrm{C}$.

$\beta=\left[\left(\beta_{m}\right)^{2}-\left(\beta_{\text {instr }}\right)^{2}\right]^{1 / 2}$

Con base en los resultados obtenidos (Tabla 2), el tamaño de cristal de $\mathrm{TiO}_{2}$ disminuyó con el aumento del porcentaje de Bi. Esto pudo estar asociado a las condiciones de preparación del material, tales como el pH (Reidy et al., 2006; Akpan et al., 2009; Nadzirah et al., 2015) así como formación de la interfaz de heterounión entre los nanocristales de $\mathrm{Bi}_{2} \mathrm{O}_{3}$ y $\mathrm{TiO}_{2}$ en el compuesto. También, se debe a que en la determinación del tamaño de cristal por medio de la Ecuación 1, el pico de difracción tiene en cuenta el efecto de las microtensiones asociadas a la inclusión del $\mathrm{Bi}_{2} \mathrm{O}_{3}$ en la matriz del $\mathrm{TiO}_{2}$. Así, al presentarse el óxido de bismuto en mayor proporción en el material, disminuye el tamaño de cristal. En este caso, los átomos de $\mathrm{Bi}$ no reemplazan los átomos de $\mathrm{Ti}$ en la estructura del $\mathrm{TiO}_{2}$, puesto que el radio de $\mathrm{Bi}^{3+}(0.096$ $\mathrm{nm})$, es mayor que el de $\mathrm{Ti}^{4+}(0.068 \mathrm{~nm})$.

Las propiedades ópticas fueron determinados mediante UV-Vis DRS. Los resultados indicaron que para el $\mathrm{TiO}_{2}$, la absorbancia en la región visible no fue significante, mientras que la reflectancia de los compósitos preparados fue mucho menor (mayor cantidad de luz fue absorbida) entre 390 y $500 \mathrm{~nm}$ debido a la contribución del $\mathrm{Bi}_{2} \mathrm{O}_{3}$ (Figura 3a). Esta condición implica la utilización de la luz visible (fotones de baja energía) en la fotorreacción. El ancho de banda se determinó mediante extrapolación de la línea recta hasta la absica de la curva (Grumes et al., 1982) a partir del gráfico de Tauc desarrollado mediante la Ecuación 3.

$\alpha(h v)=A\left(h v-E_{g}\right)^{m / 2}$

Donde $\alpha$ es el coeficiente de absorción, A es una constante de absorción, $h v$ la energía del fotón, y $m=1$ para transición directa entre bandas. Los espectros UV experimentales para las muestras preparadas de $\mathrm{Bi}_{2} \mathrm{O}_{3} / \mathrm{TiO}_{2}$ se muestran en la Figura $3 \mathrm{~b}$.

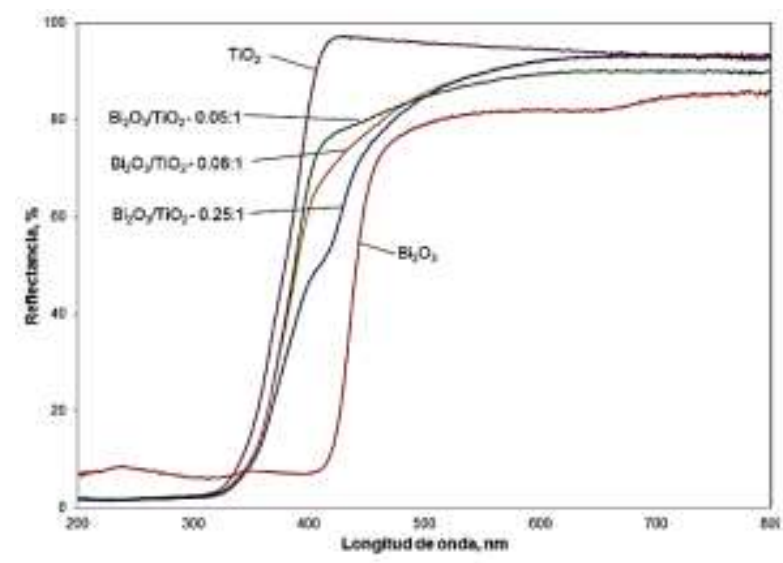

a)

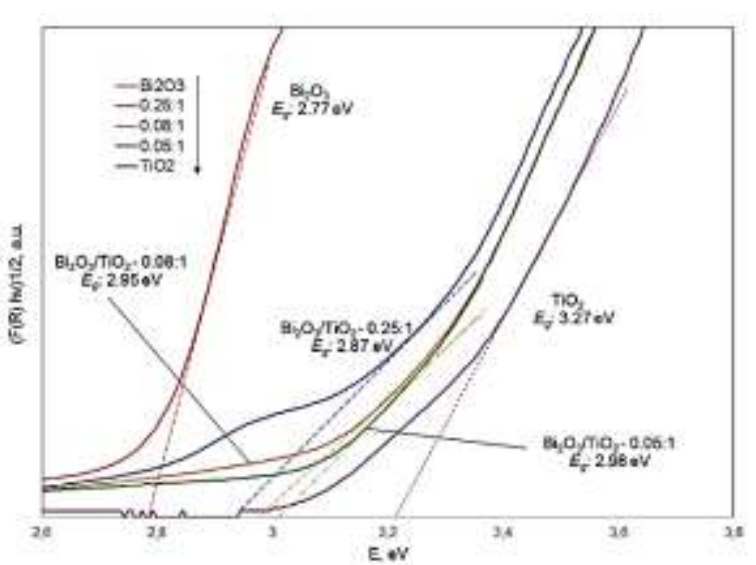

b)

Fig. 3. a) Espectro UV-Vis de los fotocatalizadores acoplados de $\mathrm{Bi}_{2} \mathrm{O}_{3} / \mathrm{TiO}_{2}$ a) Reflectancia b) Límites de absorción óptica. 
En general, se notó una disminución del ancho de banda cuando la proporción de $\mathrm{Bi}_{2} \mathrm{O}_{3}$ aumentó en el material que implica una excitación de los fotoelectrones y huecos con un mejor aprovechamiento de la luz visible. La Tabla 2 resume algunas características ópticas y físicas del sistema $\mathrm{Bi}_{2} \mathrm{O}_{3} / \mathrm{TiO}_{2}$. Estas sugieren la presencia de $\mathrm{Bi}_{2} \mathrm{O}_{3}$ con alta dispersión sobre partículas de $\mathrm{TiO}_{2}$ que impidieron el crecimiento cristalino del $\mathrm{TiO}_{2}$ durante el proceso de calcinación.

Tabla 2. Caracterización de muestras de $\mathrm{Bi}_{2} \mathrm{O}_{3} / \mathrm{TiO}_{2}$ preparadas mediante el método de impregnación húmeda $\left(\mathrm{T}_{\text {calcinación }}=300^{\circ} \mathrm{C}\right)$.

\begin{tabular}{|c|c|c|c|}
\hline Muestra & $\begin{array}{c}\text { Tamaño promedio de cristal } \\
(\mathrm{nm})\end{array}$ & $\begin{array}{c}\text { Límite de absorción } \\
(\mathrm{nm})\end{array}$ & $\begin{array}{c}\text { Ancho de banda } \\
(\mathrm{eV})\end{array}$ \\
\hline $\mathrm{Bi}_{2} \mathrm{O}_{3}$ & 61.7 & 447.59 & 2.77 \\
\hline $\mathrm{TiO}_{2}$ & 23.0 & 379.15 & 3.27 \\
\hline $0.05: 1 \mathrm{Bi}_{2} \mathrm{O}_{3} / \mathrm{TiO}_{2}$ & 20.7 & 416.05 & 2.98 \\
\hline $0.08: 1 \mathrm{Bi}_{2} \mathrm{O}_{3} / \mathrm{TiO}_{2}$ & 19.0 & 420.28 & 2.95 \\
\hline $0.25: 1 \mathrm{Bi}_{2} \mathrm{O}_{3} / \mathrm{TiO}_{2}$ & 14.3 & 432 & 2.87 \\
\hline
\end{tabular}

El límite de absorción aumentó con el incremento del $\mathrm{Bi}_{2} \mathrm{O}_{3}$ el cual fue controlado por el contenido de $\mathrm{Bi}$. El ancho de banda de energía para el $\mathrm{Bi}_{2} \mathrm{O}_{3}$ y $\mathrm{TiO}_{2}$ fueron reportados con valores 2.73 eV y 3.2 eV respectivamente (Wang et al., 2011).

Los espectros FT-IR se registraron en el intervalo de $4000-400 \mathrm{~cm}^{-1}$ con el fin de identificar los grupos funcionales del sistema $\mathrm{Bi}_{2} \mathrm{O}_{3} / \mathrm{TiO}_{2}$ (Figura 4). Las vibraciones de estiramiento del enlace $\mathrm{Bi}-\mathrm{O}$ (de oxígeno sin puente), asociadas a unidades $\mathrm{BiO}_{6}$ y $\mathrm{BiO}_{3}$ se detectaron en 630 y $845 \mathrm{~cm}^{-1}$, respectivamente (Eugen et al., 2009). La banda localizada en $1404 \mathrm{~cm}^{-1}$ aproximadamente corresponde a modo de flexión O-H (Singh et al., 2012). La banda infrarroja en $1631 \mathrm{~cm}^{-1}$ se atribuye a las vibraciones de agua libre absorbida sobre el bismuto presente en la superficie del acople $\left(\mathrm{Bi}-\mathrm{H}_{2} \mathrm{O}\right)$ (Shamaila et al., 2009). Es de observar el mayor grado de intensidad de las bandas asociadas con vibraciones $-\mathrm{OH}\left(2400\right.$ y $\left.3500 \mathrm{~cm}^{-1}\right)$ (Mozia et al., 2010).

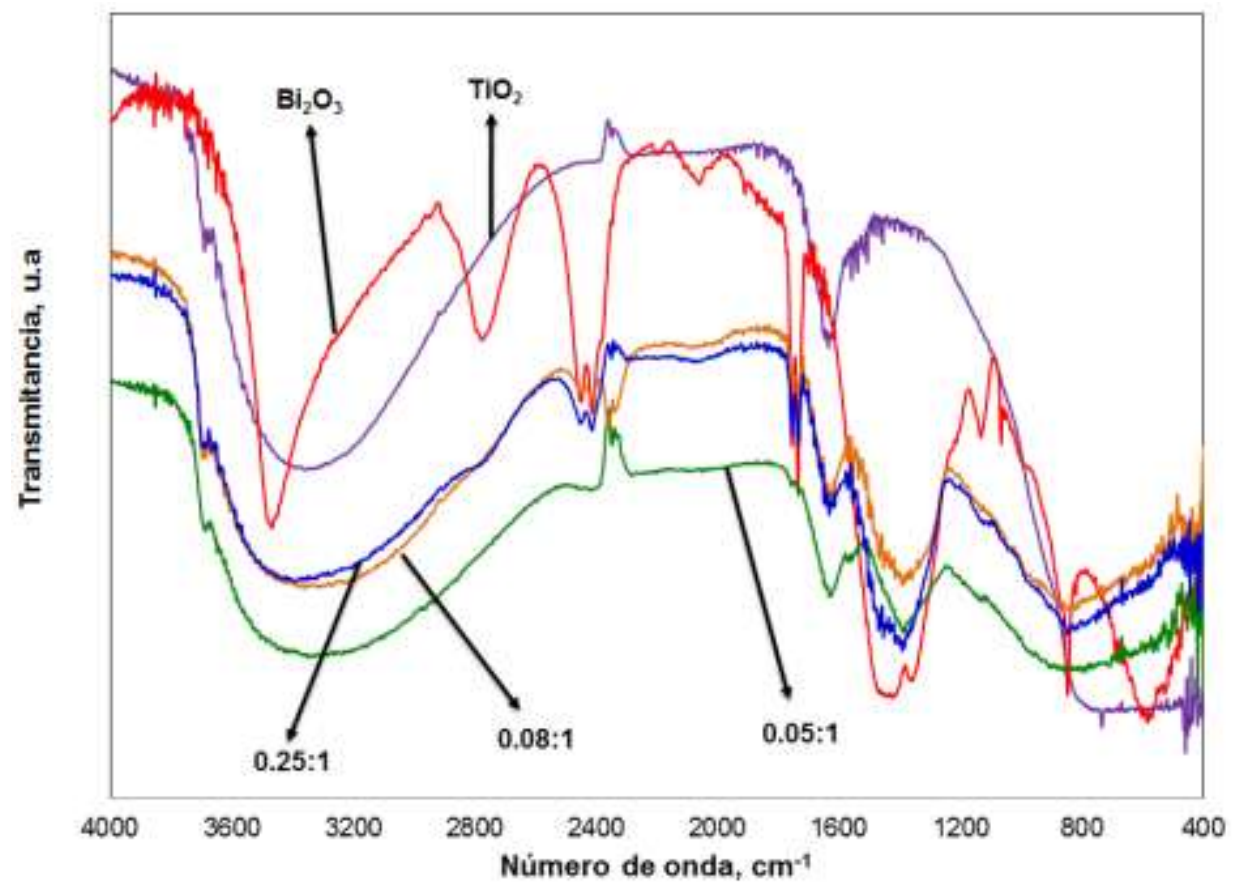

Fig. 4. Espectro FTIR de los compósitos de $\mathrm{Bi}_{2} \mathrm{O}_{3} / \mathrm{TiO}_{2}$ preparados mediante impregnación húmeda.

Se ha reconocido que los grupos superficiales hidroxilo juegan un papel importante en la reacción fotocatalítica por inhibir la recombinación del par e $/ \mathrm{h}^{+}$y producir especies activas con el oxígeno (Shamaila et al., 2009). Para este caso, el análisis FTIR confirmó que el fotocatalizador acoplado tipo compósito tiene mayor densidad de grupos $\mathrm{OH}^{-}$superficiales que el $\mathrm{TiO}_{2}$, la cual incrementó con la carga de $\mathrm{Bi}_{2} \mathrm{O}_{3}, d_{\text {debido a }}$ la deposición de Bi sobre la superficie. 


\section{Actividad fotocatalítica}

Para optimizar las condiciones de degradación de contaminantes orgánicos presentes agua residual de la industria litográfica, se utilizaron un total de 13 experimentos para modelar la superficie de respuesta (Tabla 1). El software sugirió un modelo cuadrático tanto para los porcentajes de remoción COT y DQO. La Tabla 3 y 4 muestran el ANOVA para la superficie de respuesta del modelo.

Tabla 3. ANOVA para la superficie de respuesta del modelo cuadrático para el porcentaje de remoción COT de agua residual de la industria litográfica. a Desviación estándar. b Coeficiente de variación.

\begin{tabular}{|l|c|c|c|c|c|}
\hline \multicolumn{1}{|c|}{ Fuente } & $\begin{array}{c}\text { Suma de } \\
\text { cuadrados }\end{array}$ & g. de I. & Cuadrado medio & Valor $F$ & Valor $p$ \\
\hline Modelo & 1707.31 & 5 & 341.46 & 18.41 & 0.0007 \\
\hline$x_{1}$ & 19.08 & 1 & 19.08 & 1.03 & 0.3442 \\
\hline$x_{2}$ & 147.33 & 1 & 147.33 & 7.95 & 0.0258 \\
\hline$x 1 \times 2$ & 117.37 & 1 & 117.37 & 6.33 & 0.0401 \\
\hline$x 1^{2}$ & 23.33 & 1 & 23.33 & 1.26 & 0.2990 \\
\hline$x 2^{2}$ & 1151.53 & 1 & 1151.53 & 62.10 & 0.0001 \\
\hline Residual & 129.80 & 7 & 18.54 & - & - \\
\hline Error puro & 0.39 & 4 & 0.097 & & \\
\hline Desv. Est. ${ }^{\text {a }}$ & 4.31 & - & R2 & 0.9293 & \\
\hline Media & 25.49 & - & R2 ajustado & 0.8789 & \\
\hline C.V. \%. ${ }^{b}$ & 16.89 & - & R2 predicho & 0.1205 & \\
\hline PRESS & 1615.72 & - & Precisión adec. & 13.706 & \\
\hline
\end{tabular}

Tabla 4. ANOVA para la superficie de respuesta del modelo cuadrático para el porcentaje de remoción DQO de agua residual de la industria litográfica. a Desviación estándar. b Coeficiente de variación.

\begin{tabular}{|l|c|c|c|c|c|}
\hline \multicolumn{1}{|c|}{ Fuente } & $\begin{array}{c}\text { Suma de } \\
\text { cuadrados }\end{array}$ & g. de I. & Cuadrado medio & Valor $F$ & Valor $p$ \\
\hline Modelo & 805.24 & 5 & 161.05 & 8.71 & 0.0065 \\
\hline$x_{1}$ & 58.61 & 1 & 58.61 & 3.17 & 0.1182 \\
\hline$x_{2}$ & 30.81 & 1 & 30.81 & 1.67 & 0.2378 \\
\hline$x_{1} X_{2}$ & 41.98 & 1 & 41.98 & 2.27 & 0.1756 \\
\hline$x_{1}{ }^{2}$ & 63.14 & 1 & 63.14 & 3.41 & 0.1071 \\
\hline$x_{2}{ }^{2}$ & 613.17 & 1 & 613.17 & 33.16 & 0.0007 \\
\hline Residual $_{\text {Error puro }}$ & 129.45 & 7 & 18.49 & - & - \\
\hline Desv. Est. ${ }^{\text {a }}$ & 4.03 & 4 & 1.01 & & \\
\hline Media & 4.30 & - & R2 & 0.8615 & \\
\hline C.V. \%. ${ }^{b}$ & 24.67 & - & R2 ajustado & 0.7626 & \\
\hline PRESS & 17.43 & - & R2 predicho & -0.2650 & \\
\hline
\end{tabular}

Tanto para las remociones de COT y DQO, mediante el ANOVA se determinó que los modelos son significantes con valores $F$ de 18.41 (probabilidad $0.07 \%$ debida al ruido) y 8.71 (probabilidad $0.65 \%$ debida al ruido) respectivamente además, el valor de $p<0.05$ indicó que el pH fue el término más significativo de los modelos sobre el proceso (Montgomery, 1996; A. Suarez-Escobar et al., 2015).

De los valores experimentales, se obtuvieron sendas ecuaciones polinómicas de segundo orden en términos de las variables codificadas (Ecuaciones 4 y 5 ):

\%Remoción COT $=-46.34-199.75 x_{1}+22.96 x_{2}+23.86 x_{1} x_{2}-142.35 x_{1}^{2}+20.42 x_{2}^{2}$

\%Remoción DQO = -103.58-350.12 $x_{1}+10.50 x_{2}+14.27 x_{1} x_{2}-234.18 x_{1}^{2}+14.90 x_{2}^{2}$

Donde $x_{1}$ y $x_{2}$ son los términos para los valores codificados de relación $\mathrm{Bi}_{2} \mathrm{O}_{3}: \mathrm{TiO}_{2}$ y $\mathrm{pH}$, respectivamente.

La Figura 5 muestra la correlación entre los valores experimentales y predichos para el porcentaje de degradación COT y DQO del agua residual $\left(R^{2}=0.9293\right.$ y $\left.R^{2}=0.8615\right)$ dentro del rango estudiado y se observa un aceptable ajuste de los datos considerando la naturaleza del proceso (agua real). 

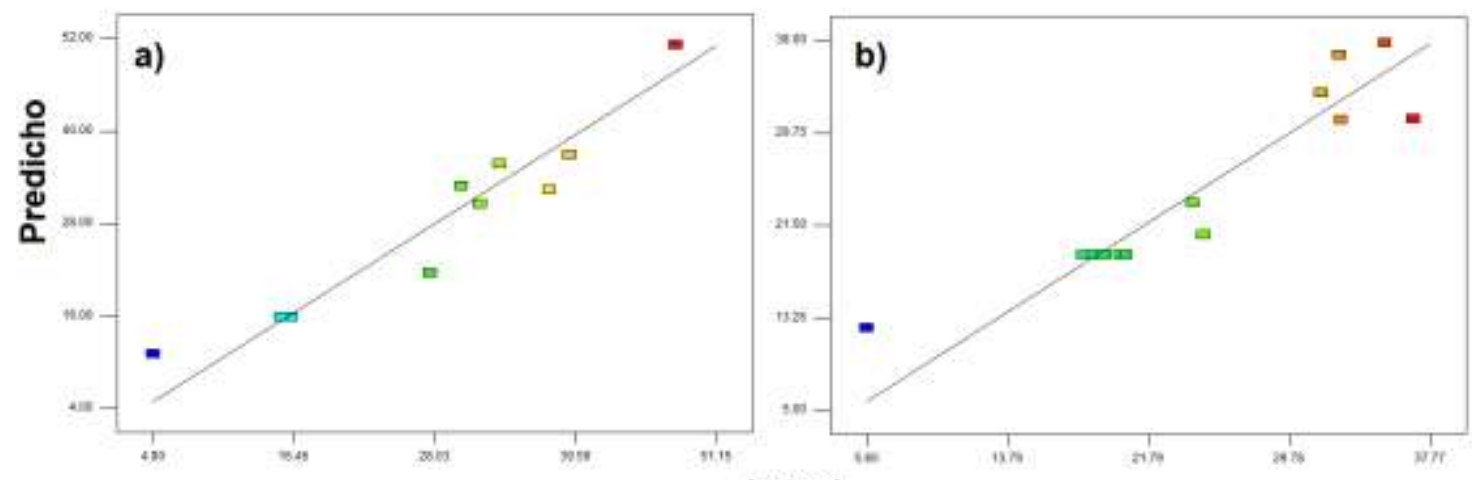

Actual

Fig. 5. Valores predichos vs. Actuales para la degradación de contaminantes orgánicos presentes en agua residual de la industria litográfica. a) Porcentaje de remoción COT. b) Porcentaje de remoción DQO.

De otra parte, se confirmó la idoneidad del modelo mediante el análisis de residuales con base en la distribución normal de los residuales (Figura 6) y el gráfico de los residuales vs. la respuesta predicha (Figura 7).

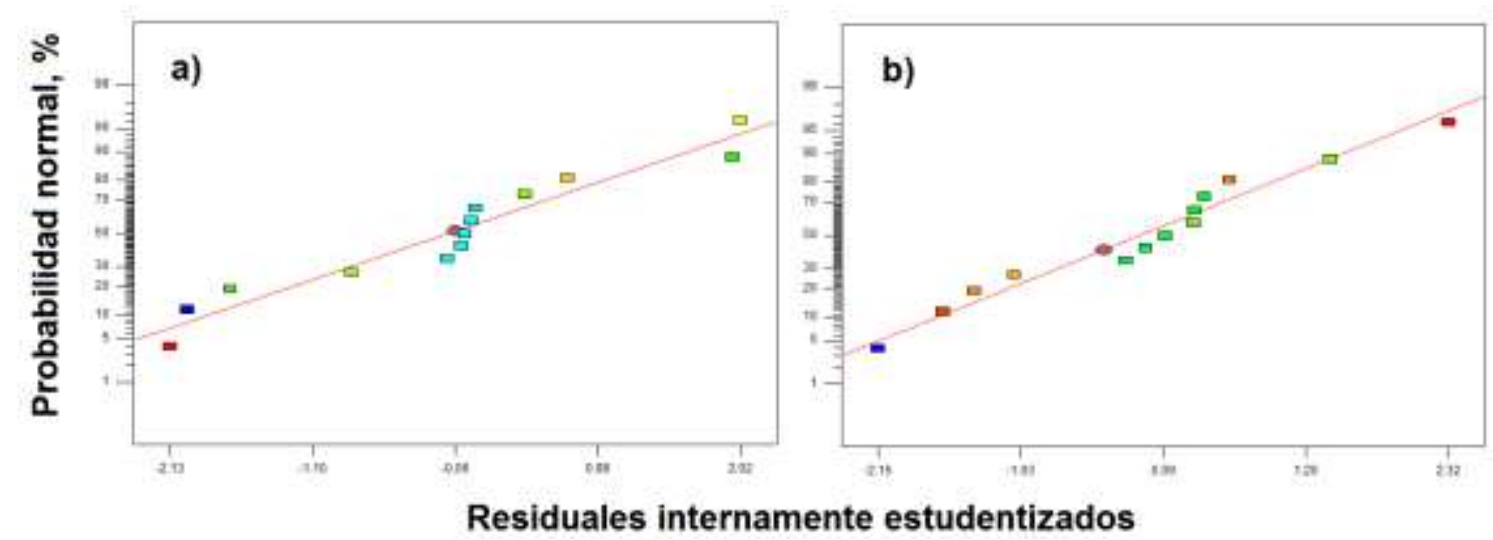

Fig. 6. Probabilidad normal de los residuales. a) Porcentaje de remoción COT. b) Porcentaje de remoción DQO.

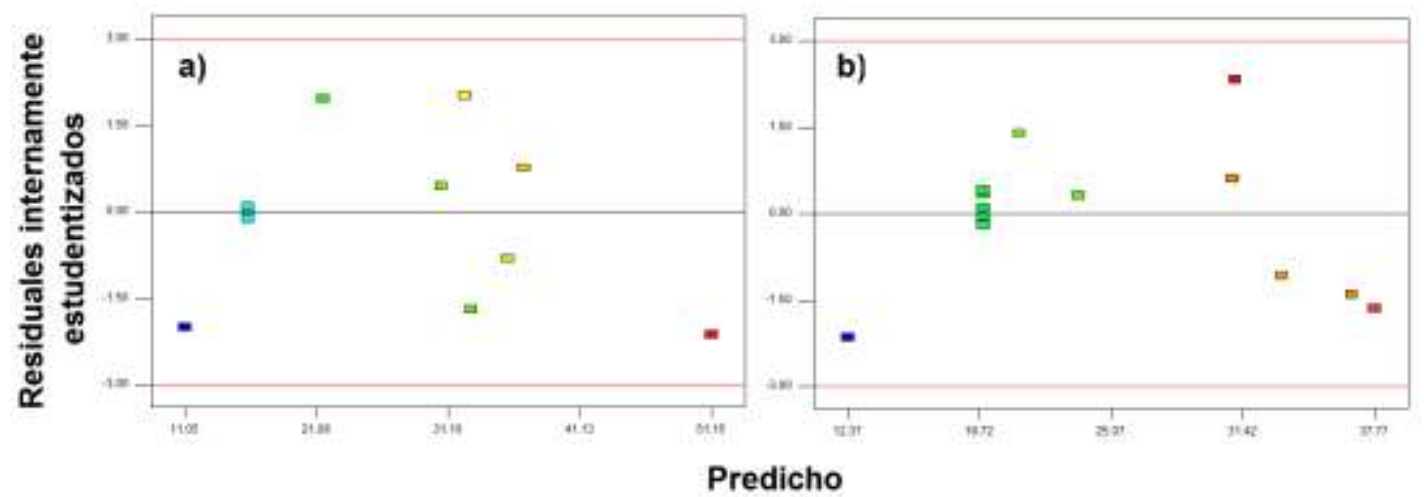

Fig. 7. Residuales vs. Respuesta predicha. a) Porcentaje de remoción COT. b) Porcentaje de remoción DQO.

Debido a que los residuales estuvieron sobre la línea recta, sugiere que los errores se encuentran normalmente distribuidos además, los patrones estructurados presentados en la distribución de los residuales vs. respuesta predicha, indican que los modelos son adecuados y no muestran ningún tipo de violación de independencia o suposición de varianza constante.

La Figura 8 muestra la influencia de la Relación $\mathrm{Bi}_{2} \mathrm{O}_{3}: \mathrm{TiO}_{2}$ y el pH sobre la degradación de contaminantes orgánicos presentes en el agua residual de la industria litográfica representada por los porcentajes de remoción COT y DQO. 
a)

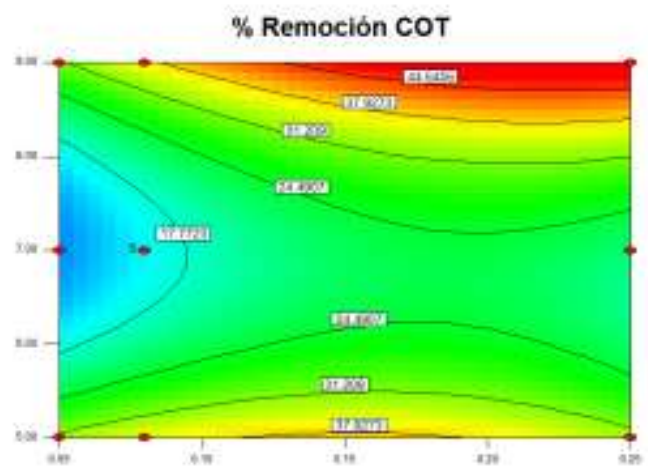

I工

b)
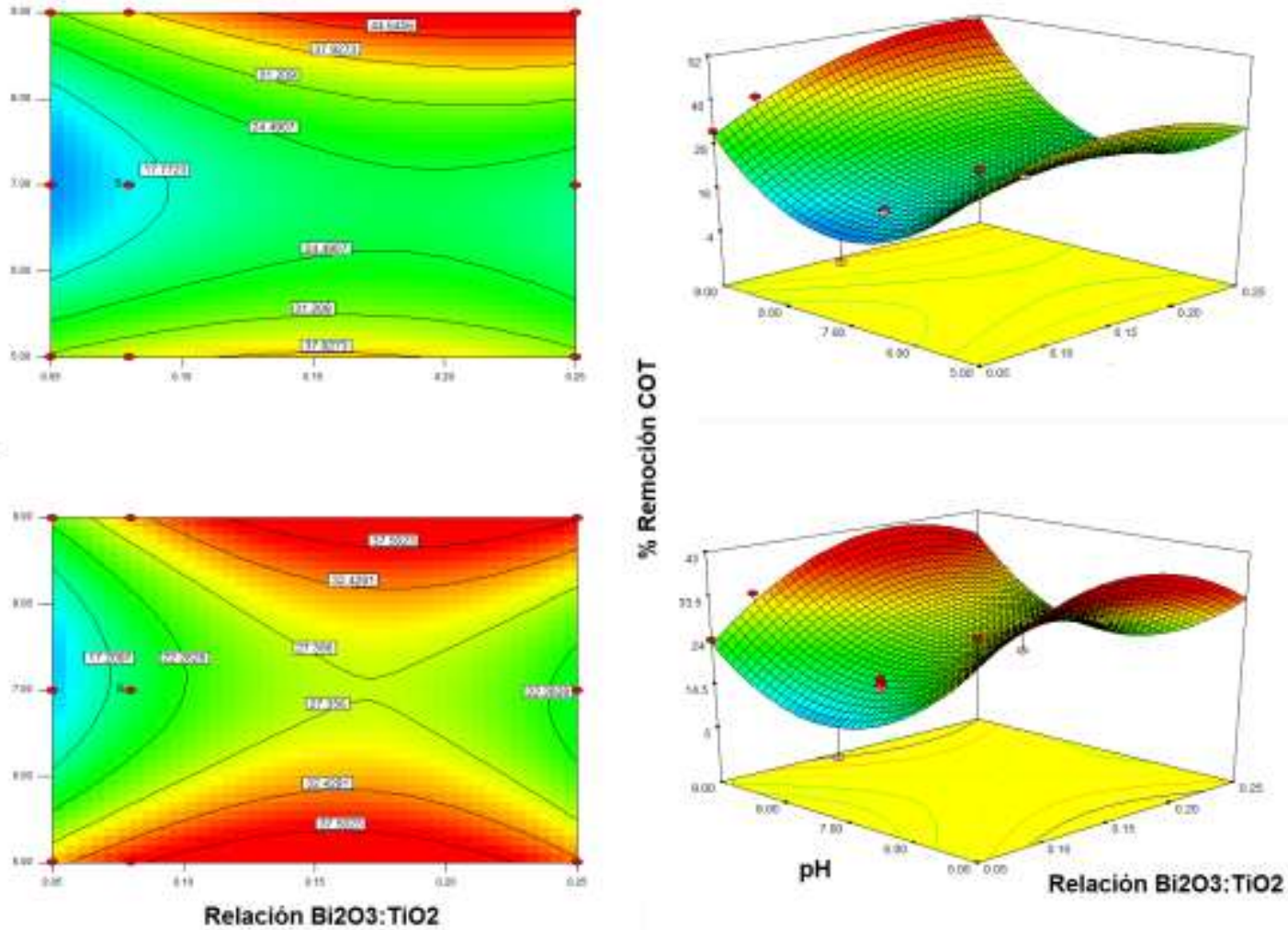

Fig. 8. Efectos de la $\mathrm{Bi}_{2} \mathrm{O}_{3}: \mathrm{TiO}_{2}$ y el pH sobre la degradación de contaminantes orgánicos presentes en agua residual de la industria litográfica. a) Porcentaje de remoción COT. b) Porcentaje de remoción DQO.

En las Figura 8, los mayores porcentajes de remoción de carga contaminante se presentan con el sistema acoplado 0.25:1.0 y pH 9.0, mientras que se pueden lograr reducciones de DQO en condiciones ácidas $(\mathrm{pH}$ 5.0) y alcalinas (pH 9.0) sin importar la relación $\mathrm{Bi} / \mathrm{Ti}$.

La Figura 9 muestra la optimización de las variables de respuesta (porcentajes de remoción COT y DQO) dentro del rango estudiado, obtenido mediante superposición de los modelos descritos por el análisis estadístico. Para el caso, las regiones resaltadas en gris muestran las zonas en donde se presentan los mayores niveles para las variables de respuesta y la intersección de las líneas de remoción COT (47.8\%) y DQO (36.8\%), muestra el punto donde se presenta la mayor remoción de carga orgánica contaminante. Con base en lo anterior, los mayores porcentajes de remoción se logran a altos niveles de relación $\mathrm{Bi}_{2} \mathrm{O}_{3}: \mathrm{TiO}_{2}$ $(0.25: 1.0)$ y $\mathrm{pH}(9.0)$.

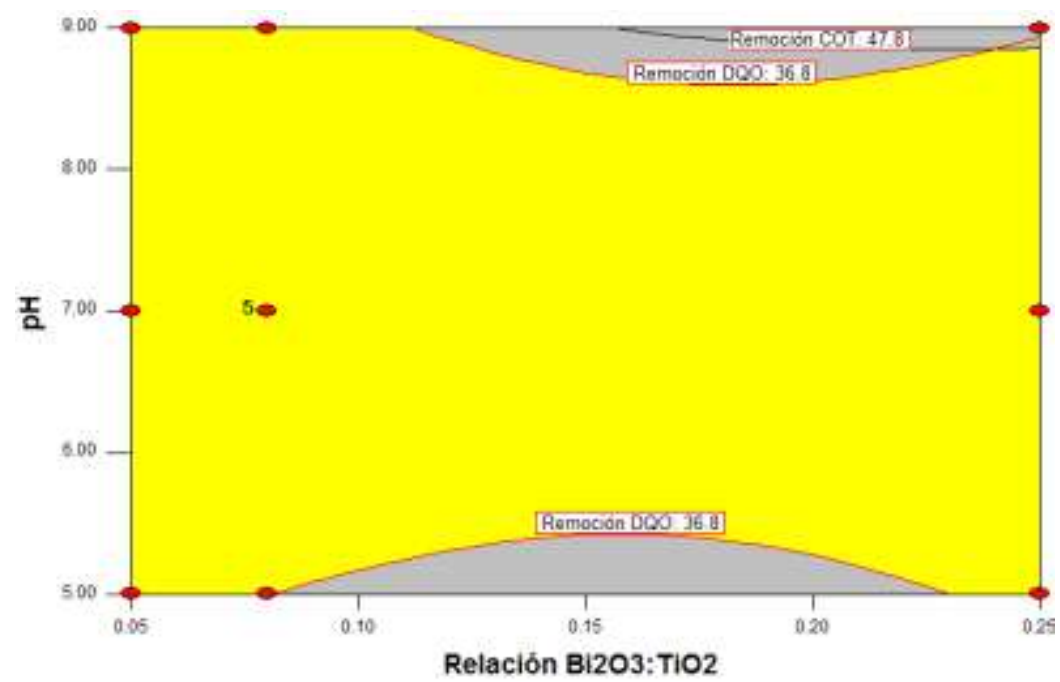

Fig. 9. Superposición de los modelos de remoción de COT y DQO para la optimización de la degradación fotocatalítica de contaminantes orgánicos presentes en agua residual de la industria litográfica. 


\section{Estudios cinéticos}

La Figura 10 muestra la cinética de degradación fotocatalítica de contaminantes orgánicos presentes en agua residual de la industria litográfica bajo irradiación UV-Visible.
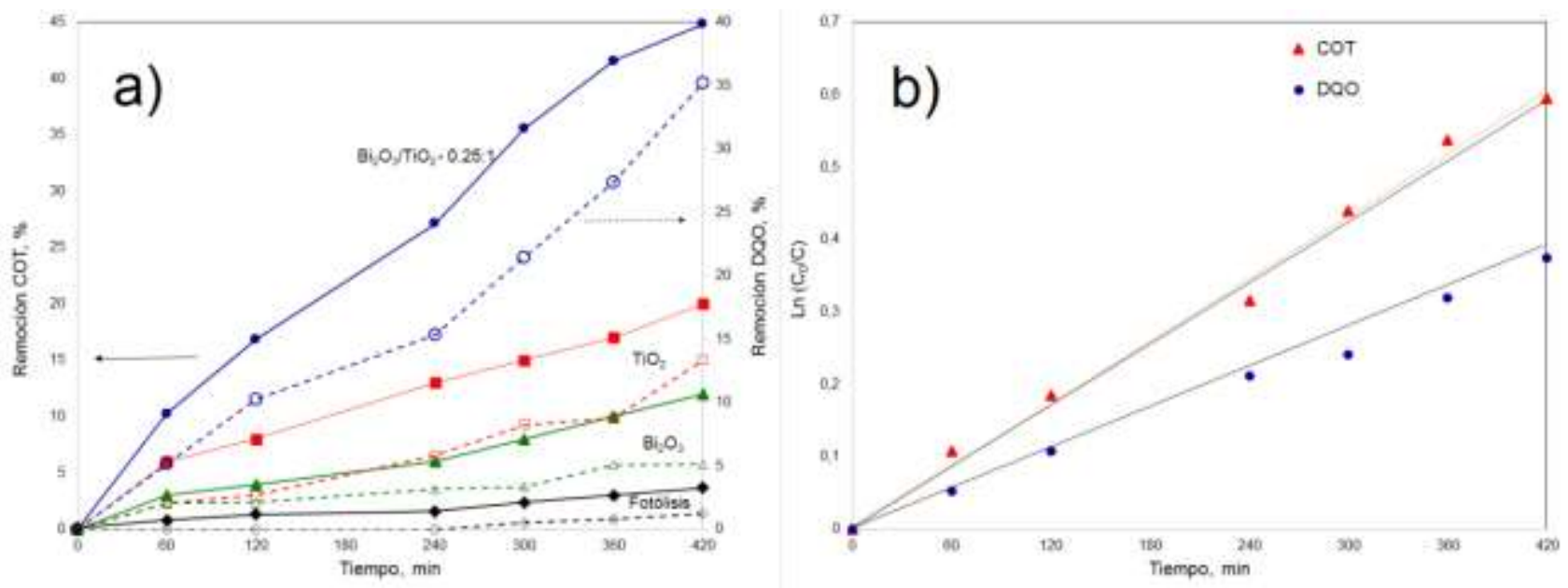

Fig. 10. a) Remoción de contenido de COT (-) y DQO (---). 0.25 g/L catalizador, pH 9.0. b) Gráfico de velocidad de primer orden para la degradación de contaminantes orgánicos por $\mathrm{Bi}_{2} \mathrm{O}_{3}$ bajo condiciones óptimas.

Mediante la determinación de la pendiente de la recta de la gráfica de $\ln \left(C_{0} / C\right)$ vs. $t$ (Fernández et al, 2002 ;. Ollis, 2005), se determinó la constante cinética de primer orden $\left(k_{1}\right)$ (Figura 8b). Los coeficientes de regresión para el modelo Langmuir-Hinshelwood (Tabla 5) implican que a pesar de tener una mezcla compleja presente en este tipo de agua residual, los resultados se pueden representar razonablemente mediante este tipo de mecanismo.

Tabla 5. Velocidad de reacción de primer orden, $k_{1}$ para la degradación fotocatalítica de contaminantes orgánicos presentes en agua de la industria litográfica.

\begin{tabular}{|c|c|c|}
\hline Modelo de remoción & Constante de velocidad de primer orden, $k_{1}\left(\times 10^{-3} \mathrm{~min}^{-1}\right)$ & $\mathrm{R}^{2}$ \\
\hline COT & 1.4 & 0.9934 \\
\hline DQO & 9.0 & 0.9954 \\
\hline
\end{tabular}

\section{CONCLUSIONES}

Se evaluó la degradación fotocatalítica de contaminantes orgánicos presentes en agua residual real de la industria litográfica mediante la utilización de un semiconductor acoplado tipo compósito de $\mathrm{Bi}_{2} \mathrm{O}_{3} / \mathrm{TiO}_{2}$ bajo irradiación UV-Visible, el cual fue preparado por el método de impregnación húmeda mediante calcinación a $300^{\circ} \mathrm{C}$. La relación 0.25:1.0 a pH 9.0 mostró la mayor actividad fotocatalítica con una remoción COT de $47.8 \%$ y DQO de $35.2 \%$. Este acople tuvo un ancho de banda de 2.87 eV (rango visible). En comparación con el $\mathrm{TiO}_{2}$, el aumento de la actividad fotocatalítica del $\mathrm{Bi}_{2} \mathrm{O}_{3} / \mathrm{TiO}_{2}$ se relacionó con la disminución del tamaño de cristal y a mayor densidad superficial de grupos hidroxilo. Se empleó un diseño experimental para desarrollar sendos modelos cuadráticos en función de los factores estudiados (relación $\mathrm{Bi}_{2} \mathrm{O}_{3} / \mathrm{TiO}_{2}$ y $\mathrm{pH}$ ), para la determinación de los porcentajes de remoción COT y DQO. La degradación fotocatalítica de compuestos orgánicos presentes en agua residual real de la industria litográfica se ajustó al modelo cinético de Langmuir-Hinshelwood tanto para la remoción de COT $\left(k_{1 \text { COT }}=1.4 \times 10^{-3} \mathrm{~min}^{-1}\right)$ como para la remoción de DQO $\left(k_{1 \mathrm{DQO}}=9.0 \times 10^{-3} \mathrm{~min}^{-1}\right)$. Además de determinar las constantes cinéticas de primer orden, con las condiciones experimentales del estudio cinético, se validaron los modelos estadísticos determinados (COT ${ }_{\text {EXP }}=44.82$ vs. COT $\mathrm{PRED}=51.15$ y DQOEXP=35.23 vs. DQOPRED=37.77) comprobando la adecuidad de los mismos.

\section{REFERENCIAS}

Akpan, U. G, B. H. Hammed. Parameters affecting the photocatalytic degradation of dyes using $\mathrm{TiO}_{2}-$ based photocatalysts: A review. Journal of Hazardous Materials: 170, 520-529 (2009)

Banisharif, A., A. A. Khodadadia, Y. Mortazavi, A. A. Firooz, J. Beheshtian, S. Agah and S. Menbari. Highly active $\mathrm{Fe}_{2} \mathrm{O}_{3}$-doped $\mathrm{TiO}_{2}$ photocatalyst for degradation of trichloroethylene in air under UV and visible light irradiation: Experimental and computational studies. Applied Catalysis B: 165, 209-221 (2015) 
Cetinkaya, T., L. Neuwirthová, K. M. Kutláková, V. Tomášek and H. Akbulut, Synthesis of nanostructured $\mathrm{TiO}_{2} / \mathrm{SiO}_{2}$ as an effective photocatalyst for degradation of acid orange. Applied surface science: 279, 384390 (2013)

Culea, E., L. Pop, M. Bosca, V. Dan,P. Pascuta, and S.Rada, Structural and physical characteristics of $x \mathrm{Gd}_{2} \mathrm{O}_{3}(100-x)\left[\mathrm{Bi}_{2} \mathrm{O}_{3} \mathrm{~B}_{2} \mathrm{O}_{3}\right]$ glasses, Journal of Physics: Conference Series: 182 (012062), 1-5 (2009)

Fernández J., J. Kiwi, C. Lizama, J. Freer, J. Baeza and H.D. Mansilla, Factorial experimental design of Orange II photocatalytic discolouration. Journal of Photochemistry and Photobiology A: 151 (1-3), 213-221 (2002)

Galeano, L, J. A. Navío, G. M. Restrepo, y J. M. Marín, Preparación de Sistemas Óxido de Titanio/Óxido de Silicio $\left(\mathrm{TiO}_{2} / \mathrm{SiO}_{2}\right)$ mediante el Método Solvotérmico para Aplicaciones en Fotocatálisis. Información tecnológica, 24(5), 81-92 (2013)

Ge, M., C. Guo, X, Zhu, L. Ma, Z, Han, W. Huand Y. Wang., Photocatalytic degradation of methyl orange using $\mathrm{ZnO} / \mathrm{TiO}_{2}$ composites. Frontiers of Environmental Science \& Engineering: 3(3): 271-280 (2009)

Grumes L.A., R.D. Leapman, C.N. Wilker, R. Hoffmann and A.B. Kuns, Oxygen K near-edge fine structure: An electron-energy-loss investigation with comparisons to new theory for selected $3 d$ Transition-metal oxides, Physical Review B: 25, 7157-7173 (1982)

Gurunathan K., Photocatalytic hydrogen production using transition metal ions-doped gamma- $\mathrm{Bi}_{2} \mathrm{O}_{3}$ semiconductor particles, International Journal of Hydrogen Energy: 29, 933-940 (2004)

Hameed A., V. Gombac, T. Montini, L. Felisri and P. Fornasiero, Photocatalytic activity of zinc modified $\mathrm{Bi}_{2} \mathrm{O}_{3}$, Chemical Physical Letters: 483, 254-261 (2009)

Hou, L.-R., C.Z. Yuan and Y. Peng. Synthesis and photocatalytic property of $\mathrm{SnO}_{2} / \mathrm{TiO}_{2}$ nanotubes composites. Journal of Hazardous Materials: 139(2), 310-315 (2007)

Ikeda A., C. Abe, T. Torimoto and B. Ohtani, Photochemical hydrogen evolution from aqueous triethanolamine solutions sensitized by binaphthol modified titanium(IV) oxide under visible-light irradiation, Journal of Photochemistry and Photobiology A: 160, 61-67 (2003)

Iliev V., D. Tomova, L. Bilyarska, L. Prahov and L. Petrov, Phthalocyanine modified $\mathrm{TiO}_{2}$ or $\mathrm{WO}_{3}$-catalysts for photooxidation of sulfide and thiosulfate ions upon irradiation with visible light. Journal of Photochemistry and Photobiology A: 159, 281-287 (2003)

Jendrucko, R.J., Coleman, Th. N. and Thomas, T.M., Waste Reduction Manual for Lithographic and Screen Printers, Tennessee, August (1994)

Li, C., J. Yuan, B. Han, L. Jian, L. and W. Shangguan, $\mathrm{TiO}_{2}$ nanotubes incorporated with CdS for photocatalytic hydrogen production from splitting water under visible light irradiation, International Journal of Hydrogen Energy: 35(13), 7073-7079 (2010)

Montgomery D.C., Design and Analysis of Experiments, 4th ed. 427-492, John Wiley \& Sons, USA, (1996)

Mozia S., A. Heciak and A.W. Morawski, Preparation of Fe-modified photocatalysts and their application for generation of useful hydrocarbons during photocatalytic decomposition of acetic acid, Journal of Photochemistry and Photobiology A: 216, 275-282 (2010).

Nadzirah, Sh., K. L. Foo and U. Hashim. Morphological reaction on the different stabilizers of titanium dioxide nanoparticles. International Journal of Electrochemical Science: 10, 5498-5512 (2015)

Ohno T., T. Mitsui and M. Matsumura, Photocatalytic activity of S-doped $\mathrm{TiO}_{2}$ photocatalyst under visible light, Chemical Letters: 32: 364-365 (2003)

Ollis, D. F., Kinetics of liquid phase photocatalyzed reactions: an illuminating approach, Journal of Physical Chemistry B: 109(6), 2439-2444 (2005)

Reidy, D. J., J. D. Holmes and M. A. Morris. The critical size mechanism for the anatase to rutile transformation in $\mathrm{TiO}_{2}$ and doped-TiO 2 . Journal of the European Ceramic Society: 26, 1527-1534 (2006) 
Shamaila, S., A. Khan, L. Sajjad, F. Chen, and J. Zhang, Study on highly visible light active $\mathrm{Bi}_{2} \mathrm{O}_{3}$ loaded ordered mesoporous titania. Applied Catalysis B: Environmental 94, 272-280 (2010)

Singh B.P., S. Nayak, S. Samal, S. Bhattacharjee and L. Besra, The role of poly(methacrylic acid) conformation on dispersion behavior of nano $\mathrm{TiO}_{2}$ powder, Applied Surface Science: 258, 3524-3531 (2012)

Suárez-Escobar, A., A. Pataquiva-Mateus andand A. López-Vasquez, Electrocoagulation-photocatalytic process for the treatment of lithographic wastewater. Optimization using response surface methodology (RSM) and kinetic study, Catalysis Today. In press (2015)

Suttiponparnit, K., J. Jiang, M. Sahu, S. Suvachittanont, T. Charinpanitkul and P. Biswas. Role of surface area, primary particle size, and crystal phase on titanium dioxide nanoparticle dispersion properties. Nanoscale Research Letters: 6(27), 1-8 (2011)

Wang L., J. Zhang, C. Li, H. Zhu, W. Wang and T. Wang, Synthesis, characterization and photocatalytic activity of $\mathrm{TiO}_{2}$ film/ $/ \mathrm{Bi}_{2} \mathrm{O}_{3}$ microgrid heterojunction, Journal of Materials Science and Technology: 27(1) 59-63 (2011)

Warren, B. E. X-Ray diffraction. 251-254, Dover Publications Inc, USA, (1968)

Yuande L., F. Xin, F. Wang, S. Luo and X. Yin, Synthesis, characterization, and activities of visible lightdriven $\mathrm{Bi}_{2} \mathrm{O}_{3}-\mathrm{TiO}_{2}$ composite photocatalysts, Journal of Alloys and Compounds: 498,179-184, (2010) 